\title{
Spin Structure Functions from Electron Scattering
}

\author{
Seonho Choi ${ }^{* \dagger}$ \\ Seoul National University \\ Seoul 151-747 Korea \\ E-mail: choi@physics.snu.ac.kr
}

The spin structure of the nucleon can play a key testing ground for Quantum Chromo-Dynamics (QCD) at wide kinematic ranges from smaller to large four momentum transfer $Q^{2}$. The pioneering experiments have confirmed several QCD sum rules at high $Q^{2}$ where a perturbative picture holds. For a full understanding of QCD at various scales, various measurements were made at intermediate and small $Q^{2}$ region and their interpretation would be a challenging task due to the non-perturbative nature. Jefferson Lab has been one of the major experimental facilities for the spin structure with its polarized electron beams and various polarized targets. A few QCD sum rules have been compared with the measured spin structure functions $g_{1}\left(x, Q^{2}\right)$ and $g_{2}\left(x, Q^{2}\right)$ at low $Q^{2}$ and surprising results have been obtained for the spin polarizabilities, $\gamma_{0}$ and $\delta_{L T}$. As for the proton spin structure functions, the lack of data for $g_{2}\left(x, Q^{2}\right)$ structure functions has been complemented with a new experiment at Jefferson Lab, SANE. The results from SANE will provide a better picture of the proton spin structure at a wide kinematic range in $x$ and $Q^{2}$.

Sixth International Conference on Quarks and Nuclear Physics,

April 16-20, 2012

Ecole Polytechnique, Palaiseau, Paris

\footnotetext{
*Speaker.

†For Jefferson Lab SANE Collaboration.
} 


\section{Introduction}

Quantum Chromo-Dynamics (QCD) governs the quarks and gluons which form the nucleons. One of the most interesting features of QCD would be the modification of the coupling constant $\left(\alpha_{S}\right)$ as a function of the scale in which the observations are made. For example, at very small scale, the coupling constant converges to zero producing so-called the asymptotic freedom, which enables the application of the usual perturbation method to the system of quarks and gluons. However, at large scale, the coupling constant gets large enough so that the perturbation calculation no longer converges, creating the non-perturbative region.

One of the important goals of modern nuclear physics is the understanding of the QCD at every scale, from perturbative to non-perturbative region. Using the dual nature of the length and momentum, the perturbative region corresponds to the high momentum scale with the nonperturbative region to the low momentum scale. Explaining the spin of the nucleon in terms of those of quarks of gluons is a key test of our knowledge of the QCD. Since it has been shown experimentally that the spin of the constituent quarks contributes only $30 \%$ to the nucleon spin (spin crisis), the study of the nucleon spin has been carried out at various laboratories in the world, probing nucleons at various momentum scales.

Most of the experiments on spin structure have used the inelastic scattering of the polarized leptons, whose kinematics can be defined as

$$
l(k)+N(p) \rightarrow l\left(k^{\prime}\right)+X
$$

with four momenta $k, k^{\prime}$ for incoming and outgoing leptons and $p$ for the nucleon target. Assuming one photon exchange during the process, the four momentum of the photon is given as $q=k-k^{\prime}$ and the momentum scale of the process is given by $q^{2}=\left(k-k^{\prime}\right)^{2}$. If we ignore the mass of the leptons with respect to their high momenta,

$$
q^{2}=\left(k-k^{\prime}\right)^{2} \simeq-2 k \cdot k^{\prime}
$$

and in the laboratory frame where the nucleon target is at rest,

$$
q^{2}=-4 E E^{\prime} \sin ^{2} \theta / 2
$$

and we define the four momentum transfer as $Q^{2}=-q^{2}$. For the lepton scattering process, $Q^{2}$ is positive.

While perturbative QCD is the natural choice at very high $Q^{2}$, in the $Q^{2}$ region covered by the various experiments, one of the most successful tools has been the formalism of the Operator Product Expansion[1, 2] (OPE) where the corresponding operators for physical processes can be expanded in terms of the increasing order of the coupling constant, $\alpha_{S}$. The OPE has produced several QCD sum rules at large momentum scale, most of which are well verified experimentally. However, in the small $Q^{2}$ region, the theoretical tools are quite limited. At exactly $Q^{2}=0$, we can rely on another QCD sum rule called Gerasimov-Drell-Hearn sum rule. Just above $Q^{2}=0$, the chiral perturbation theory $(\chi \mathrm{PT})$ can be used up to $Q^{2} \simeq m_{\pi}^{2}[3,4,5]$. In the intermediate region, lattice QCD calculations may be the only available method.

This manuscript will focus on the experimental results on the spin structure of the nucleon from inclusive electron scattering experiments, mainly using the data from Jefferson Lab $[6,7,8,9]$. 


\section{Spin Structure of the Nucleons}

With the advent of polarized electron beam and targets, the study of the nucleon spin structure enriched our understanding of Quantum ChromoDynamics (QCD), the underlying theory of the strong interaction. The spin structure of the nucleon is described in terms of two structure functions, $g_{1}\left(x, Q^{2}\right)$ and $g_{2}\left(x, Q^{2}\right)$. In the parton model, $g_{1}\left(x, Q^{2}\right)$ has a simple interpretation as unpolarized structure functions $\left(F_{1}\right.$ and $\left.F_{2}\right)$ do, but $g_{2}\left(x, Q^{2}\right)$ doesn't.

From the polarized lepton scattering experiment on the polarized nucleon target, the cross section difference for various combinations of the beam and target polarization directions produce

$$
\begin{aligned}
\frac{d^{2} \sigma}{d E^{\prime} d \Omega}(\downarrow \Uparrow-\uparrow \uparrow) & =\frac{4 \alpha^{2}}{M Q^{2}} \frac{E^{\prime}}{v E}\left[\left(E+E^{\prime} \cos \theta\right) g_{1}\left(x, Q^{2}\right)-\frac{Q^{2}}{v} g_{2}\left(x, Q^{2}\right)\right] \\
\frac{d^{2} \sigma}{d E^{\prime} d \Omega}(\downarrow \Rightarrow-\uparrow \Rightarrow) & =\frac{4 \alpha^{2} \sin \theta}{M Q^{2}} \frac{E^{\prime 2}}{E} \frac{1}{v^{2}}\left[v g_{1}\left(x, Q^{2}\right)+2 E g_{2}\left(x, Q^{2}\right)\right],
\end{aligned}
$$

where the first arrow ( $\downarrow$ or $\uparrow$ ) shows the polarization direction for the lepton beam while the second one $(\Uparrow$ or $\Rightarrow$ ) is for the target nucleon polarization.

Once $g_{1}\left(x, Q^{2}\right)$ and $g_{2}\left(x, Q^{2}\right)$ are obtained, various integrals can be considered. The most straightforward comparison can be made with the Gerasimov-Drell-Hearn (GDH) sum rule[10, 11], which is valid for the real photon $\left(Q^{2}=0\right)$. For non-zero $Q^{2}$ values as in our measurement, we can define a GDH integral as an extension. In terms of structure functions, the GDH integral can be written as

$$
I_{\mathrm{GDH}} \equiv \frac{16 \pi^{2} \alpha_{\mathrm{em}}}{Q^{2}} \int_{0}^{1}\left[g_{1}\left(x, Q^{2}\right)-\frac{4 M^{2} x^{2}}{Q^{2}} g_{2}\left(x, Q^{2}\right)\right] d x .
$$

And at $Q^{2}=0$, the GDH sum rule states

$$
I_{\mathrm{GDH}}\left(Q^{2}=0\right)=-\frac{2 \pi^{2} \alpha_{\mathrm{em}}}{M^{2}} \kappa^{2},
$$

where $\kappa$ is the anomalous magnetic moment of the target.

One of the related integral is the first moment of $g_{1}\left(x, Q^{2}\right)$ structure function,

$$
\Gamma_{1}\left(Q^{2}\right)=\int_{0}^{1} g_{1}\left(x, Q^{2}\right) d x .
$$

The difference of $\Gamma_{1}\left(Q^{2}\right)$ between the proton and the neutron at large $Q^{2}$ gives the Bjorken sum rule[12, 13], which has been verified within $10 \%$ level at large $Q^{2}$ values.

At high $Q^{2}$, where contributions of twists higher than 3 are negligible, the OPE shows that $g_{1}\left(x, Q^{2}\right)$ contains twist-2 contribution only, whereas $g_{2}\left(x, Q^{2}\right)$ contains both twist-2 and twist-3 contributions, of which the simplest case is one gluon exchange during the scattering process. The twist-3 contributions are a direct manifestation of quark-gluon interaction and quark masses. Its measurement and comparison with theory is another important test of QCD.

The higher-twist effect of $g_{2}\left(x, Q^{2}\right)$ can be evaluated by the quantity $d_{2}\left(Q^{2}\right)$,

$$
\begin{aligned}
d_{2}\left(Q^{2}\right) & =\int_{0}^{1} x^{2}\left[2 g_{1}\left(x, Q^{2}\right)+3 g_{2}\left(x, Q^{2}\right)\right] d x \\
& =3 \int_{0}^{1} x^{2} \overline{g_{2}}\left(x, Q^{2}\right) d x,
\end{aligned}
$$


where

$$
\begin{aligned}
\overline{g_{2}}\left(x, Q^{2}\right) & =g_{2}\left(x, Q^{2}\right)-g_{2}^{W W}\left(x, Q^{2}\right) \\
g_{2}^{W W}\left(x, Q^{2}\right) & =-g_{1}\left(x, Q^{2}\right)+\int_{x}^{1} \frac{g_{1}\left(y, Q^{2}\right)}{y} d y .
\end{aligned}
$$

Here $g_{2}^{W W}\left(x, Q^{2}\right)$, derived by Wandzura-Wilczek [14], is the twist-2 contribution to $g_{2}\left(x, Q^{2}\right)$.

The fact that the leading order to $g_{2}\left(x, Q^{2}\right)$ contains twist-3 contributions has another implication for the sum rule. While the Bjorken sum rule $[12,13]$ can be re-derived using the OPE, a similar sum rule for $g_{2}\left(x, Q^{2}\right)$ cannot.

The sum rule for $g_{2}\left(x, Q^{2}\right)$ reads

$$
\int_{0}^{1} g_{2}\left(x, Q^{2}\right) d x=0
$$

It was first derived by Burkhardt and Cottingham (BC) [15] in 1969, based on the dispersion relation and the asymptotic behavior of the corresponding Compton amplitude. As mentioned earlier, the $\mathrm{BC}$ sum rule can not be confirmed with the OPE [16]. On the other hand, vanishing higher-twist effects do imply the BC sum rule.

At $Q^{2}$ values close to zero, two quantities are of particular interest: generalized spin polarizabilities, $\gamma_{0}$ and $\delta_{L T}$, defined as

$$
\begin{aligned}
\gamma_{0}\left(Q^{2}\right) & =\frac{16 M^{2} \alpha_{\mathrm{em}}}{Q^{6}} \int_{0}^{x_{0}} x^{2}\left\{g_{1}\left(x, Q^{2}\right)-\frac{4 M^{2} x^{2}}{Q^{2}} g_{2}\left(x, Q^{2}\right)\right\} d x \\
\delta_{L T}\left(Q^{2}\right) & =\frac{16 M^{2} \alpha_{\mathrm{em}}}{Q^{6}} \int_{0}^{x_{0}} x^{2}\left\{g_{1}\left(x, Q^{2}\right)+g_{2}\left(x, Q^{2}\right)\right\} d x,
\end{aligned}
$$

where $x_{0}$ denotes the exclusion of the elastic scattering kinematics at $x=1$. Due to the additional $x^{2}$ weight, the contribution to the integral from the small $x$ region is very small, which is well suited to the experiment.

\section{Facilities}

The measurement of the two spin structure functions $g_{1}\left(x, Q^{2}\right)$ and $g_{2}\left(x, Q^{2}\right)$ requires both a polarized lepton beam and polarized nucleon targets. With the advanced techniques for polarized beam and targets, there have been a series of experiments to measure the spin structure functions of the proton and the neutron from DESY, CERN, SLAC and Jefferson Lab. Most of the these experiments have used polarized electron beams with an exception to CERN where polarized muon beams were used.

Jefferson Lab provides $6 \mathrm{GeV}$ polarized electron beams with about $80 \%$ polarization. Available targets at JLab include polarized proton/deuteron target using frozen $\mathrm{NH}_{3}$ of $\mathrm{ND}_{3}$ and polarized ${ }^{3} \mathrm{He}$ target as an effective polarized neutron target. Among the three experimental halls, Hall-B with CLAS detector has the largest coverage but the direction of the target polarization is limited to only longitudinal direction. The other two halls, Hall-A and Hall-C, using conventional magnetic spectrometers, do not have this constraint and can use transversely polarized targets. 
The polarized ${ }^{3} \mathrm{He}$ target in Hall-A can achieve about $60 \%$ polarization with a luminosity up to $10^{36} \mathrm{~cm}^{2} \mathrm{~s}^{-1}$. Polarized $\mathrm{NH}_{3}$ or $\mathrm{ND}_{3}$ targets can have about 70 to $90 \%$ (proton) or 30 to $40 \%$ (deuteron) polarization and their luminosity is limited to $10^{35} \mathrm{~cm}^{2} \mathrm{~s}^{-1}$ (Halls A and C) and $10^{34} \mathrm{~cm}^{2} \mathrm{~s}^{-1}$ (Hall-B).

\section{Spin Structure Functions and QCD Sum Rules}

\subsection{Spin Structure Functions and Various Integrals}

During the last decade, there has been a significant progress for spin structure functions both for the proton and the neutron. In this manuscript, a small fraction of data, mostly at low $Q^{2}$, will be presented. Most of the presented data were obtained from Jefferson Lab. Figure 1 summarizes various integrals of spin structure functions. The integral of $g_{1}\left(x, Q^{2}\right), \Gamma_{1}$ is available for very wide range of $Q^{2}$ and it is now possible to study its evolution from the perturbative to the nonpertubative regime. On the other hand, for $g_{2}\left(x, Q^{2}\right)$, more data are available for the neutron than for the proton. As shown in the figure, the integral $\Gamma_{2}$ shows a reasonable satisfaction of the $\mathrm{BC}$ sum rule for the neutron and ${ }^{3} \mathrm{He}$, while there is a sizable violation for the proton at $Q^{2}=5(\mathrm{GeV} / c)^{2}$. A major contribution to the $d_{2}$ integral comes from $g_{2}\left(x, Q^{2}\right)$ and that explains the lack of data for the proton. For the neutron, the evolution of $d_{2}$ agrees reasonably well with the phenomenological model MAID. More data for the neutron is expected at much smaller $Q^{2}$.

The measured spin polarizabilities are one of the most interesting results available from JLab. The experimental results agree quite well with the MAID estimation while NNLO $\chi$ PT calculations are not compatible with the experimental results. It is especially surprising for $\delta_{L T}$ since generally large contributions from the $\Delta$ resonance are expected to cancel out for $\delta_{L T}$. To resolve the issue, a new experiment has been completed at Jefferson Lab to measure the spin polarizabilities for the proton target at very low $Q^{2}$.

\section{$4.2 g_{2}\left(x, Q^{2}\right)$ Structure Function for the Proton}

As seen from Fig. 1, the data for $g_{2}\left(x, Q^{2}\right)$ structure function for the proton is quite scarce because the measurement involves rotating the proton target polarization perpendicular to the electron beam polarization. This is the major motivation for a new experiment at Jefferson Lab, Spin Asymmetries on the Nucleon Experiment (SANE)[17]. The SANE experiment uses the polarized, frozen $\mathrm{NH}_{3}$ target with a new non-magnetic spectrometer to cover a wide range of $Q^{2}$. Figure 2 shows a schematic view of the setup. The new non-magnetic spectrometer is mainly composed of Cerenkov detector for particle identification and BigCal calorimeter for energy measurement.

With large solid angle of about $200 \mathrm{msr}$ and two electron beam energies at 4.7 and $5.9 \mathrm{GeV}$, the SANE experiment covers a quite large kinematic range: $2.5<Q^{2}<6.5(\mathrm{GeV} / c)^{2}$ and $0.3<x<0.8$. Figure 3 shows the kinematical coverage of SANE for perpendicular configuration superposed over the existing measurements.

The invariant mass $W$ has been maintained above $1.4 \mathrm{GeV} / c^{2}$. With the proton target polarization of about $71 \%$ in both parallel and almost perpendicular with respect to the electron beam, the experiment can measure both $g_{1}\left(x, Q^{2}\right)$ and $g_{2}\left(x, Q^{2}\right)$ without any external input for $g_{2}\left(x, Q^{2}\right)$. The existing spectrometer, HMS of Hall-C of Jefferson Lab has served to measure target parameters 

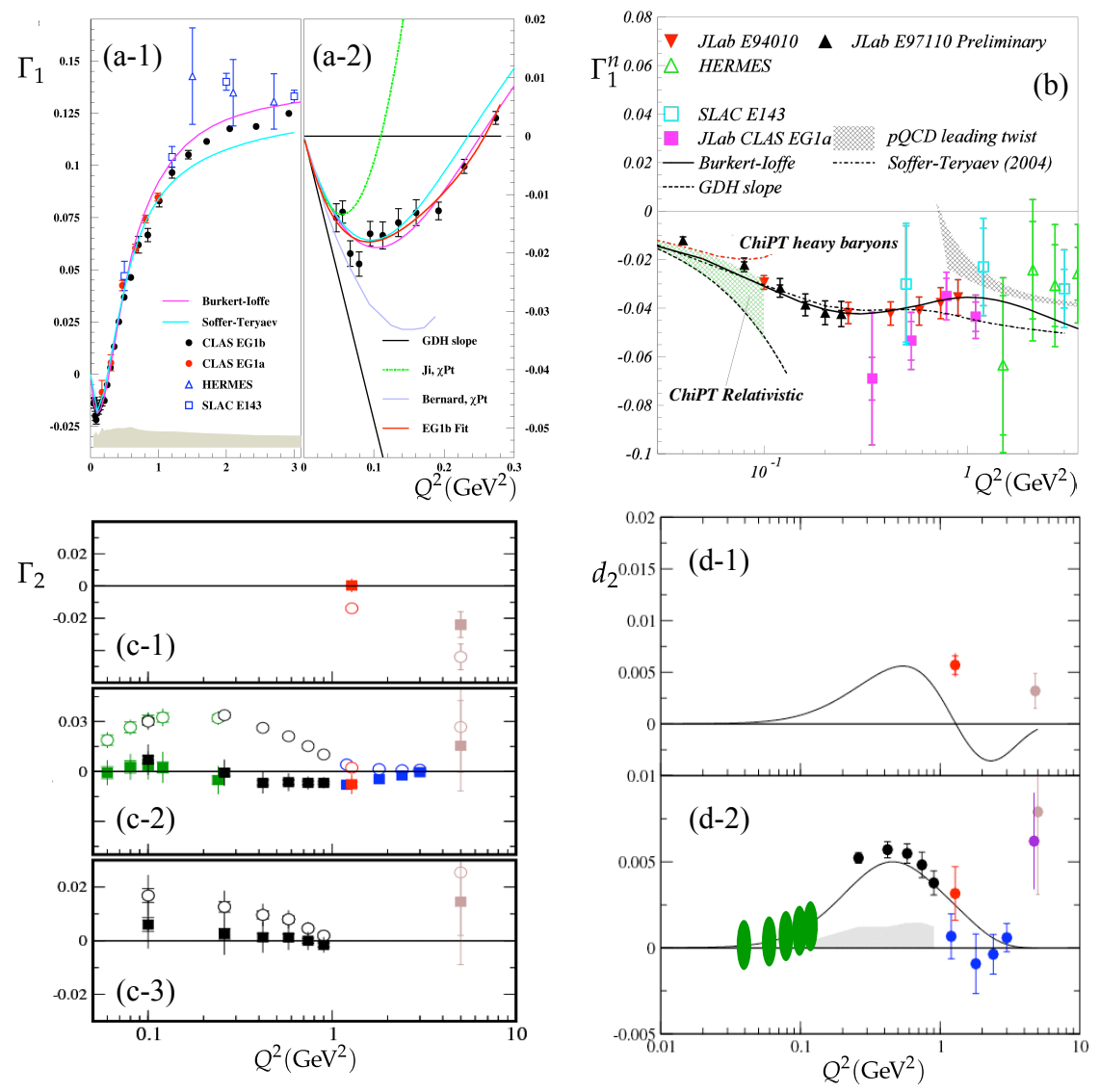

Figure 1: Various integrals of spin structure functions. (a-1) Integral of $g_{1}\left(x, Q^{2}\right)$ for the proton (a-2) Integral of $g_{2}\left(x, Q^{2}\right)$ for the neutron at small $Q^{2}$ region (b) Evolution of $\Gamma_{1}$ for the neutron as a function of $Q^{2}$ (c-1) Integral of $g_{2}\left(x, Q^{2}\right)$ for Burkhardt-Cottingham sum rule for the proton (c-2) $\Gamma_{2}$ for the neutron (c-3) First measurement of $\Gamma_{2}$ on nuclear target, ${ }^{3} \mathrm{He}$. For (c-1), (c-2) and (c-3) open symbols are for the integrals of $g_{2}\left(x, Q^{2}\right)$ over resonance region only and filled symbols include estimates of the non-measured DIS region. (d-1) $d_{2}$ integral for the proton (d-2) $d_{2}$ integral for the neutron.

such as packing fraction and to have an independent measurement of asymmetries for a few limited kinematic conditions.

The new measurement will provide high quality data for $g_{2}\left(x, Q^{2}\right)$ structure function for the proton over the previously unmeasured region. From its coverage in $x$ and $Q^{2}$, it is possible to study the evolution of the structure function as a function of $Q^{2}$. The data at constant $Q^{2}$ will contribute to the integral of the structure functions such as Burkhardt-Cottingham sum rule or $d_{2}$ of the proton. The experiment was successfully completed and the data analysis is in progress and a few preliminary results were presented at this conference during the talk.

\section{Summary}

Since the spin crisis, progress has been made for spin structure of the nucleon over the past decades. Now it is possible to have a global picture of the spin structure at various scales of $Q^{2}$. 

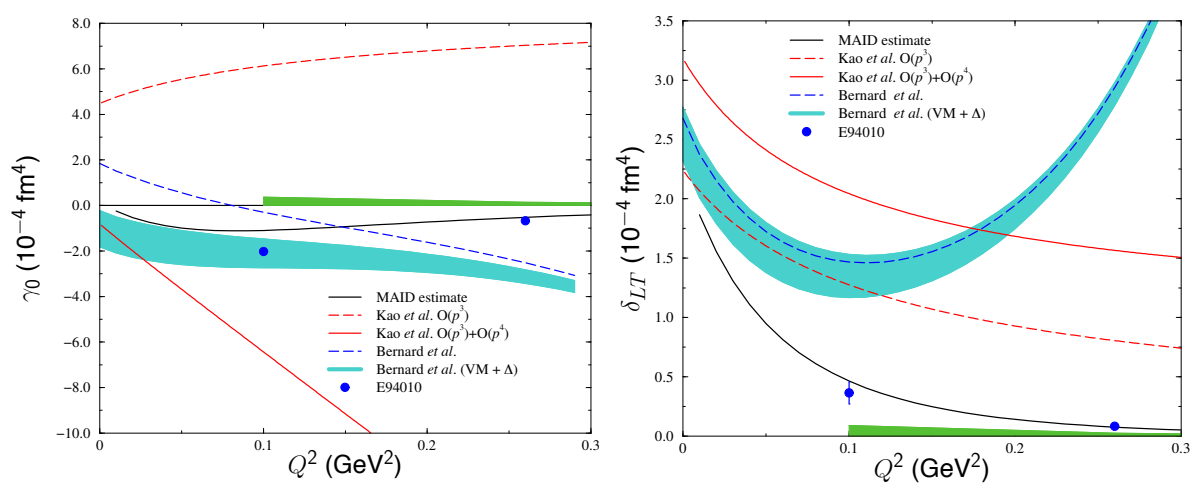

Figure 2: Spin polarizabilities for the neutron at low $Q^{2}$. The solid red line represents chiral perturbation calculation up to $O\left(p^{4}\right)$, which is at odds with both measurements for $\gamma_{0}$ and $\delta_{L T}$. The data is in a reasonable agreement with the phenomenological model MAID.

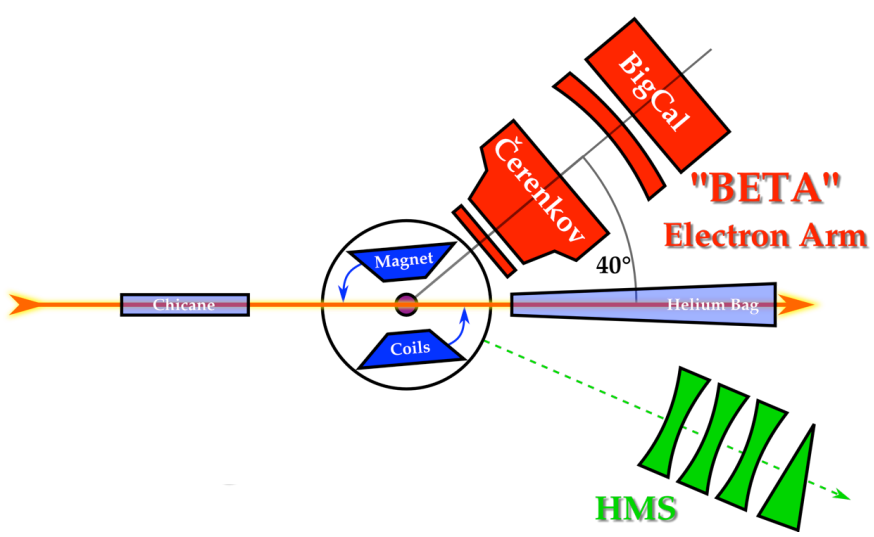

Figure 3: Schematic view of the SANE setup in Hall-C of Jefferson Lab. Chicane was necessary to compensate the deflection of the beam by the target magnet. The target magnet can be rotated for $0^{\circ}$ or $80^{\circ}$ with respect to the beam. Non magnetic spectrometer BETA is composed mainly of Cerenkov detector and $\mathrm{BigCal}$ calorimeter. After the target, $\mathrm{He}$ bag has been installed to further reduce the background to BETA spectrometer. The existing HMS was used for measuring beam parameter and for an independent measurement of asymmetries at limited kinematic conditions.

While SLAC, CERN, DESY provide most of the data at high $Q^{2}$, Jefferson Lab has been successful in measuring spin structure functions for intermediate to low $Q^{2}$ region. Spin structure functions for the neutron have been another notable contribution from Jefferson Lab.

If high $Q^{2}$ data has confirmed several QCD sum rules thanks to the perturbative interpretation of the QCD, with non-perturbative feature of the QCD, the low $Q^{2}$ region can play an interesting testing ground for a full understanding of QCD over the entire kinematic range. In this regard, with its high polarization electron beam at $6 \mathrm{GeV}$ and various polarized targets, Jefferson Lab has been playing a major role for measuring the spin structure of the nucleon at low $Q^{2}$. The $g_{1}\left(x, Q^{2}\right)$ spin structure function shows a spectacular change of the GDH integral from large $Q^{2}$ toward the large and negative GDH sum rule value at $Q^{2}=0$, while the $g_{2}\left(x, Q^{2}\right)$ spin structure function shows some hints of the Burkarhdt-Cottingham sum rule. The effect of higher-twists has been 


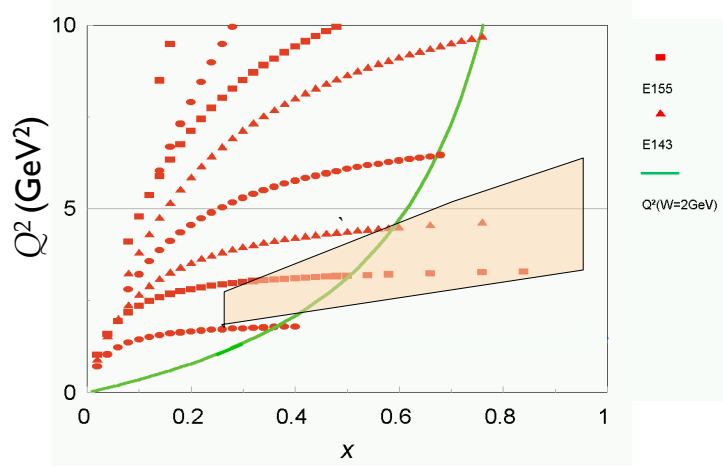

Figure 4: Kinematical coverage of SANE for the configuration for perpendicular beam and target polarization. The filled area is for SANE providing new data for the proton $g_{2}\left(x, Q^{2}\right)$ structure function in the mid $Q^{2}$ range.

studied with $d_{2}$ integral coming from $g_{2}\left(x, Q^{2}\right)$ structure function. Interesting results are obtained for the spin polarizabilities where the most elaborate $\chi \mathrm{PT}$ calculations are still incompatible with the experimental results. A new experiment to resolve this mystery by measuring the proton spin structure functions at even smaller $Q^{2}$ region was completed and the final results will soon be available.

As for $g_{2}\left(x, Q^{2}\right)$ structure function, data for the proton target is less than those for the neutron and the new experiment at Jefferson Lab, SANE, tries to fill the gap in this regard. SANE experiment uses an almost perpendicularly polarized proton target and measures $g_{2}\left(x, Q^{2}\right)$ and $g_{1}\left(x, Q^{2}\right)$ structure functions over a wide range of kinematics. The data will provide the first glimpse of the full picture of $g_{2}\left(x, Q^{2}\right)$ structure function of the proton. The data analysis is in the final stage and a few preliminary results were presented at this conference during the talk.

\section{References}

[1] E. Shuryak and A. Vainshtein, Nucl. Phys. B201, 141 (1982).

[2] R. L. Jaffe and Xiangdong Ji, Phys. Rev. D 43, 724 (1991).

[3] D. Drechsel, S. Kamalov and L. Tiator, Phys. Rev. D 63, 114010 (2001).

[4] X. Ji and J. Osborne, J. Phys. G 27, 127 (2001).

[5] V. Bernard, T. Hemmert and Ulf-G. Meissner, Phys. Lett. B 545, 105 (2002).

[6] M. Amarian et al., Phys. Rev. Lett. 89, 242301 (2002).

[7] M. Amarian et al., Phys. Rev. Lett. 92, 022301 (2004).

[8] M. Amarian et al., Phys. Rev. Lett. 93, 152301 (2004).

[9] J.-P. Chen, A. Deur and F. Garibaldi, JLab proposal E97-110, The GDH Sum Rule and the Spin Structure of ${ }^{3}$ He and the Neutron using Nearly Real Photons.

[10] S. B. Gerasimov, Sov. J. Nucl. Phys. 2, 430 (1966).

[11] S. D. Drell and A. C. Hearn, Phys. Rev. Lett. 16, 908 (1966). 
[12] J. D. Bjorken, Phys. Rev. 148, 1467 (1966).

[13] J. D. Bjorken, Phys. Rev. D 1, 1376 (1970).

[14] S. Wandzura and F. Wilczek, Phys. Lett. B72, 195 (1977).

[15] Hugh Burkhardt and W. N. Cottingham, Annals of Physics 56, 453 (1970).

[16] M. Anselmino, A. Efremov and E. Leader, Phys. Rep. 261, 1 (1995); Erratum-ibid, 281399 (1997).

[17] S. Choi, M. Jones, Z.-E. Meziani, O. Rondon, JLab Proposal E07-003, Spin Asymmetries on the Nucleon Experiment. 